ene

\title{
INNOVACIONES QUE PUEDEN \\ CAMBIARLO TODO: EL FUTURO DE LA ENFERMERÍA QUIRÚRGICA
}

\section{INNOVATIONS THAT CAN CHANGE EVERYTHING: THE FUTURE OF SURGICAL NURSING}

\author{
José Manuel Asencio Gutiérrez
}

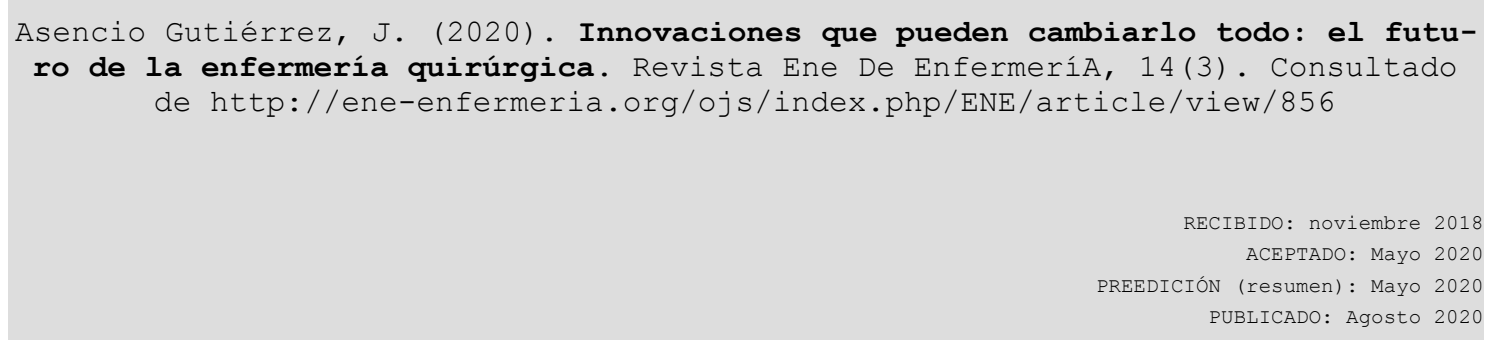




\section{Resumen}

La posibilidad de recoger y estructurar datos de forma masiva en los bloques quirúrgicos supone un salto cualitativo en la capacidad de generar nuevos conocimientos y algoritmos. Esta revisión literaria recopila innovaciones recientes en el entorno quirúrgico en relación con la mejora de los recursos materiales y humanos, de la seguridad del paciente y de las comunicaciones, incorporando distintas visiones sobre el impacto de este nuevo contexto para la Enfermería Quirúrgica.

Palabras claves: tecnología; innovación organizacional; automatización; tendencias; enfermería de quirófano; enfermería quirúrgica

\section{Abstract}

The possibility of collecting and structuring data massively in the surgical blocks represents a qualitative leap in the ability to generate new knowledge and algorithms. This new context, in addition to requiring Nursing greater capacity for technological adaptation, could mean the transformation of their professional role. This literary review describes recent innovations in the surgical environment in relation to the improvement of material and human resources, patient safety and communications. Surgical nursing should adapt its role claiming the care of psychosocial care, a task that is not susceptible to be automated.

Keywords: technology, organizational innovation, automation, trends, operating room nursing, perioperative nursing. 


\section{INTRODUCCIÓN}

En sus notas "Qué es y qué no es la enfermería" (1859), Florence Nightingale (1820-1910), considerada hoy precursora de la Enfermería profesional, resaltó la enorme importancia de la observación para los cuidados. Esta observación no es otra cosa que la recopilación, el estudio y la interpretación de datos; en otras palabras, resaltaba la importancia de introducir el método científico para mejorar los cuidados. (1)

Nightingale, que tenía amplios conocimientos en estadística, los aplicó en epidemiología para mejorar los estándares de salud en un contexto histórico complejo, ya que sus primeros pasos coinciden con el inicio de la Primera Revolución Industrial, proceso que cambió rápida y radicalmente la sociedad a todos los niveles. (1)(2)

El contexto histórico hoy sitúa a la enfermería en otro punto de inflexión debido a una nueva Revolución Industrial. Ésta supondrá una profunda transformación en la generación y distribución de energía, en movilidad y en comunicación (2). Las nuevas herramientas para la recogida y estructura de datos de forma masiva suponen un salto cualitativo en la capacidad de generar nuevos conocimientos y algoritmos (3). La observación de la que hablaba Nightingale tiene hoy un potencial como nunca antes.
Por la naturaleza de la actividad quirúrgica y por la alta tecnología asociada al funcionamiento de los quirófanos, es esperable que se produzcan modificaciones sustanciales en las labores asistenciales de Enfermería. Desde nuestra perspectiva temporal no podemos despejar la incertidumbre en torno al futuro de los cuidados perioperatorios debido a que las innovaciones que están por venir generarán sinergias poco predecibles entre ellas, como ocurre hoy, por ejemplo, entre el teléfono móvil y los nuevos sistemas de pago.

Para la enfermería quirúrgica resulta de interés conocer las últimas tendencias en la implantación de innovaciones tecnológicas y organizacionales relacionadas con los cuidados perioperatorios, para adaptarse a las exigencias de la profesión en un futuro inmediato.

\section{Objetivo}

Conocer las innovaciones que están teniendo lugar en el entorno quirúrgico para analizar su impacto en la enfermería.

\section{Metodología}

Se opta por una revisión de la literatura relacionada con el tema planteado, utilizando las siguientes bases de datos, repositorios web o metabuscado- 
res: PubMed, CUIDEN Plus, Cochrane

Plus, CINAHL y Portal Regional de la BVS.

Se utilizaron descriptores en

Ciencias de la Salud (DeCS) para las búsquedas en castellano y términos MeSH (Medical Subject Headings) para búsquedas en inglés. (Tabla 1)

Tabla 1: Tesauros utilizados en la búsqueda

\begin{tabular}{|c|c|}
\hline Español & Inglés \\
\hline & \\
Tecnología (DeCS) & Technology (MeSH) \\
Tecnología biomédica (DeCS) & Biomedical technology (MeSH) \\
Innovación organizacional (DeCS) & Inventions (MeSH) \\
Automatización (DeCS) & Organizational innovation (MeSH) \\
Tendencias (DeCS) & Automation (MeSH) \\
& Trends (subheading MeSH) \\
\hline Enfermería perioperatoria (DeCS) & \\
Enfermería de quirófano (DeCS) & Operating room nursing (MeSH) \\
\end{tabular}

La búsqueda tuvo lugar durante el mes de junio de 2018. Todas las búsquedas fueron delimitadas mediante filtros a artículos publicados entre los años 2013 y 2018.
En la siguiente tabla se describen las cadenas de búsqueda exactas utilizadas en cada una de las fuentes documentales. 
Tabla 2: Estrategia de búsqueda

\begin{tabular}{|c|c|}
\hline Fuente & Cadena de búsqueda (y observaciones) \\
\hline$P_{L}$ & $\begin{array}{l}\text { (Technology OR Biomedical technology OR Organizational innovation OR Inventions } \\
\text { OR Organizational innovation OR Automation OR Trends) AND (Operating room } \\
\text { nursing OR perioperative nursing) }\end{array}$ \\
\hline \multirow{2}{*}{$\begin{array}{c}\text { Cuiden } \\
\text { Plus }\end{array}$} & $\begin{array}{l}\text { [cla="Tecnología"] or [cla="Tecnología sanitaria"] or [cla="Innovación"] and } \\
\text { [cla="Enfermería quirúrgica"] and [cla="Enfermería perioperatoria"] }\end{array}$ \\
\hline & $\begin{array}{l}\text { Búsqueda por palabras clave. Se realizó una segunda búsqueda porque Tendencias } \\
\text { no aparece en la lista de palabras clave de Cuiden Plus: Tendencias AND } \\
\text { (enfermería quirúrgica OR enfermería perioperatoria). }\end{array}$ \\
\hline CINAHL & $\begin{array}{l}\text { (Technology OR Biomedical technology OR Organizational innovation OR Inventions } \\
\text { OR Organizational innovation OR Automation OR Trends) AND (Operating room } \\
\text { nursing OR perioperative nursing) }\end{array}$ \\
\hline $\begin{array}{c}\text { Cochrane } \\
\text { Plus }\end{array}$ & $\begin{array}{l}\text { (TECNOLOGÍA) OR (TECNOLOGÍA SANITARIA) OR (INNOVACIÓN) OR } \\
\text { (TENDENCIAS) AND (ENFERMERÍA QUIRÚRGICA) OR (ENFERMERÍA } \\
\text { PERIOPERATORIA) }\end{array}$ \\
\hline \multirow{2}{*}{$\begin{array}{l}\text { Portal } \\
\text { Regional } \\
\text { de la BVS }\end{array}$} & $\begin{array}{l}\text { ((tw:(Tecnología)) OR (tw:(Tecnología sanitaria)) OR (tw:(Innovación)) OR (tw: } \\
\text { (innovación organizacional)) OR (tw:(automatización)) OR (tw:(tendencias))) AND } \\
\text { ((tw:(Enfermería perioperatoria)) OR (tw:(Enfermería quirúrgica))) }\end{array}$ \\
\hline & $\begin{array}{l}\text { Búsqueda avanzada. Se activó el filtro “Asunto de la revista: Enfermería”, quedando } \\
\text { delimitada la búsqueda a MEDLINE y LILACS. }\end{array}$ \\
\hline
\end{tabular}

Los criterios utilizados fueron establecidos transversalmente para todas las bases de datos consultadas.

Criterios de inclusión:

Artículos originales, revisiones sistemáticas o literarias, editoriales u opiniones de expertos, en inglés y español.
Artículos disponibles online a texto completo.

Criterios de exclusión:

Artículos relativos a innovaciones en técnicas quirúrgicas.

Artículos sobre el papel de la enfermería en la cirugía robótica. 
En Tabla 3 se presenta un resumen de los artículos incluidos en la revisión.

\section{RESULTADOS}

En la siguiente figura se resumen los resultados de la búsqueda y el pro- ceso de selección de los artículos para la revisión. (Figura 1)

Los artículos incluidos fueron clasificados en tres categorías: eficiencia en el flujo de trabajo, calidad de los cuidados y rol de enfermería.

Figura 1: Estrategia de selección de artículos

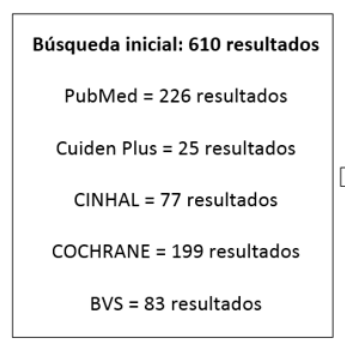

\section{DISCUSIÓN}

La Asociación de Enfermeras Perioperatorias de los Estados Unidos de América (AORN), en colaboración con un grupo de investigación de enfermería informática de Chamberlain College of Nursing (Illinois, EE.UU.), desarrolló en 2015 la herramienta ATOR (AORN Technology in the Operation Room) para clasificar la tecnología utilizada en quirófano. Esto permitió catalogar cada producto según los efectos percibidos en Eficiencia en el Flujo de Trabajo (Workflow Efficiency) y en Calidad de los cuidados del paciente (Quality Patient Care). (4)

El concepto Eficiencia en el Flujo de Trabajo hace alusión a cómo se tra-

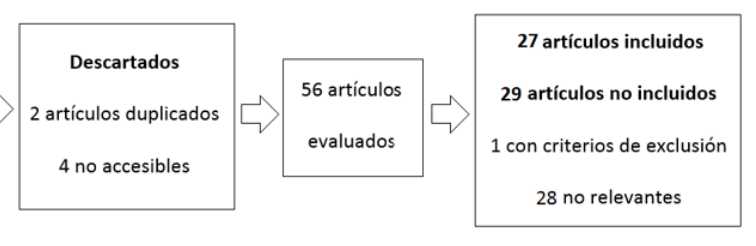

baja, en relación con el tiempo y los recursos disponibles. Es un concepto para el que no hay uso consensuado (5), por lo que en adelante utilizaremos rendimiento como sinónimo.

La Calidad de los Cuidados del Paciente refleja el impacto que producen en los indicadores las tecnologías utilizadas en sanidad. Actualmente hay poca evidencia de correlación entre el uso de las tecnologías de la información y mejoras específicas de los resultados en salud en EE.UU., pero sí está demostrada su existencia entre estas y la calidad clínica (5).

Estas dos variables de resultados de la herramienta ATOR resultan útiles para presentar los resultados de la revisión llevada a cabo. Aunque es frecuente 
que las innovaciones que aumentan el rendimiento produzcan a su vez un impacto indirecto en la calidad de los cuidados, y viceversa (4), la percepción subjetiva de las mismas permite su clasificación. Esta no debe tomarse en ningún caso como otra cosa que una forma más o menos ordenada de estructurar la información.

Innovaciones que suponen una mejora potencial del rendimiento

Los artículos consultados sugieren que las innovaciones relacionadas con la mejora del rendimiento en los bloques quirúrgicos tienen que ver con la optimización del trabajo a través de cambios organizacionales (con o sin análisis de grandes volúmenes de datos), con la prevención de riesgos laborales y con el entrenamiento a través de simulaciones.

La importancia de la recolección e interpretación de datos para mejorar los cuidados comenzó a mediados del siglo XIX con el trabajo de Florence Nightingale (1)(6). Desde entonces, la capacidad de extracción de datos ha sido automatizada en gran parte, permitiendo como nunca la recopilación de información, que puede ser analizada y utilizada para mejorar la seguridad del paciente y los resultados clínicos, mejorar la gestión de los recursos humanos y materiales, reducir ineficiencias, optimizar procesos y volumen de trabajo, y establecer prioridades e iniciativas. (6)

La analítica descriptiva aplicada a la historia clínica digital está permitiendo entornos perioperatorios con cada vez más ítems que pueden ser medidos, y por tanto, mejorados a través de la identificación de patrones y datos sobre comportamiento y técnicas. (6)

Dewes (6) identifica siete puntos clave que determinan la calidad de los servicios de cirugía: costes excesivos, comienzos sin retraso de la cirugía, tasa de cancelación de procedimientos, retrasos de los ingresos en unidades de cuidados posanestésicos, beneficio que genera horariamente el paciente (ítem orientado a servicios privados), tiempo de rotación del paciente y tendencia de la predicción de la duración del procedimiento. Estos ítems van a tender a centrar la atención de los gestores para mejorar los servicios.

El objetivo de recolectar y analizar datos en el entorno perioperatorio es la transformación de estos en información útil para facilitar los cambios en la dirección adecuada. Por ejemplo, permitir al personal conocer semanalmente los datos de rotación del servicio puede inspirar una sana competición para reducir los tiempos. En ese sentido, Azzi et al. (7) llevaron a cabo un estudio piloto que concluía que, flexibilizando 30 minutos la 
jornada laboral, más de la mitad de las lobectomías podrían haber acabado a su hora al evitar cambios de turno. Sus autores sugieren que estas jornadas flexibles, aplicadas con respeto a la normativa laboral, mejoraría la seguridad del paciente a la vez que ofrecería un ambiente de trabajo saludable para el equipo. Esto mejoraría los resultados del hospital ya que, el funcionamiento del área quirúrgica tiene un impacto directo en el funcionamiento general, tanto en lo operativo como en lo financiero, y está relacionado con la satisfacción y la seguridad de los pacientes (7).

El análisis de datos aplicado a la programación de los quirófanos permite optimizar los tiempos, manteniendo cierto margen para atender cirugías de emergencia (6). Esto está teniendo un impacto positivo en el rendimiento del bloque quirúrgico.

Guedon et al. (8) desarrollaron un sistema para predecir el final de las colecistectomías laparoscópicas, con la intención de identificar a tiempo real el mejor momento para empezar a preparar al próximo paciente. Instalaron un sensor en el electrobisturí para monitorizar su uso. Con los datos recopilados en 57 intervenciones reconocieron un patrón que les permitió determinar que el momento óptimo para ello era 25 minutos antes de la última sutura. Posteriormen- te, se comparó la capacidad del sistema para identificar ese momento, con la de los responsables de la cirugía. El sistema predictivo resultó bastante más preciso que el personal sanitario en cirugías de más de 40 minutos, consiguiendo un margen de error absoluto más bajo en comparación.

Por su parte, Kranzfelder et al. (9) aportaron un sistema de detección a tiempo real del material quirúrgico en uso, basado en tecnología de identificación por radiofrecuencia (más conocida por sus siglas en inglés, RFID). Etiquetaron 110 instrumentos quirúrgicos habituales en cirugías laparoscópicas, permitiendo en cada momento conocer aquellos que estaban siendo utilizados. Esta innovación permite un análisis mucho más profundo, basado en datos, del flujo de trabajo de cirujanos e instrumentistas durante el acto quirúrgico, convirtiendo en medible lo que antes no lo era.

Las innovaciones que reducen los riesgos profesionales también tienen un impacto en el rendimiento del factor trabajo en los bloques quirúrgicos. McGlozlin y Cole (10) aplicaron los recientes avances en espectrofotometría infrarroja para cuantificar escapes de gases anestésicos en una unidad de recuperación posanestésica, evidenciando que el personal de enfermería está expuesto a niveles significativos de N2O y sevofluo- 
rano en unidades poco ventiladas. Su trabajo demuestra, además, la efectividad de las mascarillas equipadas con tecnología de absorción de gases para evitar su difusión en el entorno del paciente.

En este sentido, Kloeze et al. (11) proponen reducir la exposición del equipo quirúrgico a otro riesgo laboral, las radiaciones. Pusieron a prueba la efectividad de unas gasas especiales con capacidad para absorber radiación, reportando una reducción significativa de la exposición (en torno a la mitad) en distintas áreas corporales de cirujanos e instrumentistas. Los autores advierten de su utilidad en procedimientos endovasculares asociadas al uso de fluoroscopia.

Diversas experiencias innovadoras en la gestión de los recursos humanos en el área quirúrgica han resultado positivas, y tienen potencial para ser generalizadas. Weller et al. (12) estudiaron la costoefectividad de invertir un programa de simulación para mejorar la formación y coordinación en 20 equipos quirúrgicos. Al coste habitual de otros cursos para el personal sanitario, 50.000 dólares neozelandeses (unos $29.000 €$ ), el programa mejoró la comunicación entre los miembros del equipo y consiguió reducir un $16 \%$ los eventos adversos.

La formación a través de simulaciones es muy usada y está extendida en los entrenamientos de técnicas quirúrgicas, pero no aún en enfermería. Glaser et al. (13) reportaron recientemente la utilidad de este método aplicado a la instrumentación quirúrgica. La reproducción del lugar de trabajo a través de un software con capacidad interactiva, desarrollado por el propio equipo investigador desde el punto de vista de la enfermería, ha dado buenos resultados en la familiarización del personal más novel con los instrumentos quirúrgicos y con su disposición en la mesa.

En EE.UU., la tecnología también ha sido utilizada para mejorar la asistencia a los pacientes críticos postquirúrgicos de su ejército, en zonas donde las plantillas pueden ser muy limitadas para las demandas que tienen que cubrir. El trabajo de VanGent et al. (14) describe el impacto inicial de introducir la asistencia médica a través de videoconferencia de un intensivista con acceso a historias clínicas, pruebas de imagen e informes de laboratorio. Tras reportar buenos resultados a nivel local, el modelo fue extendido a hospitales militares a $4.500 \mathrm{~km}$ de distancia. Los autores piensan que el modelo debe expandirse más allá de los hospitales militares al otro lado del océano, ya que permitiría mejorar la capacidad asistencial en barcos o en campos de batalla. Es evidente el potencial que tiene este modelo en la atención del 
paciente rural, la valoración inicial de pacientes quemados, el triage de víctimas y en la asistencia en transporte aéreo.

Los trabajos anteriormente mencionados perfilan en conjunto una nueva etapa, que supone un cambio cualitativo por su capacidad transformadora del rendimiento en el entorno de trabajo. Generalmente, la enfermería quirúrgica percibe que la tecnología orientada a mejorar el rendimiento del trabajo en el quirófano tiene menos valor que aquella que mejora directamente la calidad de los cuidados (4), pero no debemos olvidar que esta clasificación es subjetiva, porque en la práctica ambos conceptos suelen estar relacionados directa o indirectamente.

Innovaciones que suponen una mejora potencial de la calidad de los cuidados

Los artículos consultados destacan la mejora de las comunicaciones, tanto en el equipo quirúrgico como entre profesionales y pacientes, o con sus familiares. Este punto parece clave a la hora de mejorar la calidad de la asistencia.

En los últimos años se han ido consolidando prácticas que, aunque no son del todo novedosas, han ido acumulando evidencia a favor y suponen un cambio sustancial por su impacto en la satisfacción de usuarios. Es el caso de la apertura de las Unidades de Recuperación Postanestésicas a las visitas de los familiares de los pacientes pediátricos (15) y la generalización de la práctica pielcon-piel tras las cesáreas (16) (que consiste en facilitar la transición de los recién nacidos al entorno extrauterino no interrumpiendo el contacto directo con la madre). Ambas, además de mejorar resultados clínicos, humanizan la asistencia y mejoran la percepción de la experiencia durante la estancia hospitalaria.

La mejora de las comunicaciones en el ámbito quirúrgico no necesariamente requiere de grandes avances tecnológicos. Wieck et al. (17) describen que el uso de un busca para enviar mensajes de texto a los familiares del paciente que está siendo intervenido les tranquiliza durante la espera. Mensajes predefinidos como "Todo va bien, acabamos de comenzar el procedimiento", "Los médicos aún están trabajando. Todo va bien" o "Hemos terminado. Vuelva a la recepción para hablar con el doctor" han demostrado capacidad para mejorar la satisfacción general, también en aquellas cirugías que sufren retrasos por alguna razón.

El intercambio de mensajes de texto entre el equipo quirúrgico y los usuarios es potencialmente útil para la educación sanitaria antes y después del ingreso para la cirugía. Hansen (18), una 
enfermera de la Universidad de San Francisco, está estudiando actualmente el impacto que puede tener esta práctica en la tasa de reingresos. De obtener resultados significativos, parece probable la generalización de experiencias parecidas, debido a su bajo coste. Es una práctica que podría ser un buen complemento para el nuevo modelo de atención Perioperative Surgical Home, que está siendo impulsado por anestesistas para el cuidado de pacientes quirúrgicos de manera integral, coordinada y a menor coste (19).

Además de las mejoras en comunicación, se están desarrollando iniciativas para mejorar la seguridad del paciente quirúrgico a través del análisis de datos clínicos.

Con motivo de mejorar la tasa de infección de heridas quirúrgicas, Esser et al. (20) se propusieron reducir un $10 \%$ la apertura de puertas de los quirófanos de un hospital de St. Paul (Minnesota, EE.UU). Para ello analizaron las necesidades de desplazamientos del personal, instalando contadores para recopilar información detallada (incluyendo el momento y el tiempo que permanecen abiertas las puertas). Tras el análisis de los motivos que causaban los desplazamientos, establecieron un plan formativo y mejoraron las comunicaciones internas, consiguiendo sobrepasar el objetivo inicial. Se redujeron un $13 \%$ los desplazamientos, aunque por el momento se desconoce el impacto en la tasa de infecciones.

El análisis de los datos ha sido útil también para el trabajo de Tepas et al. (21) Este equipo fue más allá de su mera interpretación, creando un indicador específico para el riesgo de complicaciones postoperatorias en intervenciones de colon a través de la compilación automática de la información recogida en la historia clínica digital. Este indicador estaba permanentemente actualizado y utilizaba como fuente de datos las observaciones de enfermería, las constantes vitales registradas y los resultados de laboratorio. Sus autores confían en la utilidad de este sistema para mejorar la calidad de la asistencia y la reducción del coste por intervención. Cabe destacar que la implantación de este innovador sistema no supuso mayor carga de trabajo en el servicio.

En esta misma línea, Levchenko et al. (22) desarrollaron un algoritmo que, gracias a la monitorización a tiempo real de los desplazamientos del personal de enfermería y del uso de los dispensadores de antiséptico de base alcohólica, consigue detectar y notificar a enfermería mediante señales visuales el momento ideal para la higiene de manos. Esta 
aumentó significativamente desde su implantación.

Además del uso de algoritmos de asistencia para la toma de decisiones clínicas, está en desarrollo otro tipo de mejoras para la seguridad del paciente quirúrgico. Soysal et al. (23) sugieren el uso de métodos de calentamiento activo con fibras de carbono para prevenir hipotermias no planificadas.

Contra la retención accidental de material quirúrgico hay diversos métodos en estudio. Una revisión sistemática reciente revela que actualmente se están utilizando protocolos que combinan el contaje manual con el uso de rayos $\mathrm{X}$, tecnología de radiofrecuencia y también material marcado con códigos de barras (24). El estudio resalta que el contaje manual es irreemplazable. Schnock et al. confirmaron posteriormente la eficacia del uso de radiofrecuencia para reducir los casos de retención de material quirúrgico (25).

Aunque las innovaciones anteriormente mencionadas pudieran ser de gran utilidad, Rao et al. inciden en la posibilidad de mejorar los resultados clínicos sin necesidad de nuevos recursos materiales, y publicaron recientemente que aquellos hospitales que permiten mayor autonomía a enfermería, tienen menor tasa de mortalidad a los 30 días en pacientes quirúrgicos. (26)
Es previsible que todas estas tecnologías e iniciativas tengan un efecto positivo en la seguridad de los pacientes, e incluso en los costes de la asistencia. ¿Pero qué transformaciones puede sufrir el rol de la enfermería de quirófano como consecuencia?

Cambios en el rol profesional

La adaptación de la profesión enfermera a los cambios que están sucediendo y aquellos que están por venir es objeto de debate entre autores.

Probablemente Luck y Gillespie sean las más pesimistas al respecto, al ofrecer una visión de futuro casi completamente orientada al trabajo técnico. Prevén una disminución de la necesidad de las habilidades profesionales de Enfermería, que en algunos casos se reduciría a la mera gestión de máquinas, e incluso sugieren que el papel de la profesión en el quirófano puede verse limitado a preparar asépticamente al paciente y a cubrir la cama. Las autoras ven posible la mejora y generalización de la cirugía robótica, de las plataformas de simulación para la formación del personal, el uso de marcadores visuales para diferenciar áreas sanas y neoplásicas, e incluso la introducción de cintas de transporte para el traslado de pacientes. Presentan la posibilidad futura de una total sustitución del personal de quirófano, a excepción de los cirujanos. (27) 
El resto de los trabajos consultados discrepan de la visión de estas dos autoras, y coinciden en que están surgiendo nuevas oportunidades para la profesión enfermera.

Odom, Rao y Chard están de acuerdo en que la enfermería está aún por encontrar la integración total en los equipos interdisciplinarios (19)(26)(28). Nuevos modelos de atención requieren de una práctica avanzada y con mayor autonomía para obtener mejores resultados (19).

Resulta llamativo que, para algunos profesionales expertos, las innovaciones que están por venir supondrán una experiencia quirúrgica diferente para los pacientes, pero de sus testimonios no se deduce la percepción de un cambio significativo en la composición del equipo quirúrgico o de la manera de trabajar. Varios expertos entrevistados imaginan nuevos dispositivos de telemonitorización, trócares inteligentes, entrenamientos virtuales, o incluso analgesia no adictiva, pero su visión refleja, en general, que no comparten la idea de una rápida y profunda transformación del entorno quirúrgico. Colleen Mattioni (responsable de los cuidados perioperatorios del Hospital Universitario de Pennsylvania), sin embargo, está convencida de que el rol de la enfermería quirúrgica está a una década del gran cambio. Ella piensa que la cirugía mínimamente invasiva y la telecirugía sacarán a los cirujanos de los quirófanos, y que los días de la enfermería generalista en el área quirúrgica están contados. Su opinión no parece mayoritaria. (29)

Enfermería tiene la capacidad y el deber de contribuir con su aportación en la configuración de los sistemas sanitarios, según el Institute of Medicine (National Academy of Sciences, Washington DC, EE.UU.) en su segundo informe sobre la profesión enfermera (28). La enfermería, lejos de avanzar hacia la obsolescencia, como señalaban Luck y Gillespie (27), está aún por ganar presencia e importancia. La falta de publicaciones del colectivo enfermero sobre cambios en los que ya está participando activamente, como el desarrollo del mencionado modelo Perioperative Surgical Home, dificulta la visibilidad de los nuevos roles de la enfermería (19). Odom invita a sus colegas de profesión a avanzar en el rol investigador.

Uno de los puntos más novedosos en los que coinciden varios autores es en el rol de la enfermería en la gestión de los datos, denominada enfermería informática en algunos países (4). La ingente cantidad de datos relativos a la salud que se generan a diario requiere de nuevas figuras. Byrne pone sobre la mesa posibles conflictos debido a la ve- 
racidad de los datos, de cara a demandas y reclamaciones entre pacientes, aseguradoras y hospitales privados. Ve en la enfermería la solución para garantizar su autenticidad. Destaca también la necesidad de que Enfermería cuide de la calidad de los datos que generan las estancias hospitalarias. El exceso de "ruido", o datos autogenerados sin utilidad clínica, podría limitar la utilidad de la tecnología en la asistencia, por lo que es conveniente su filtrado para priorizar lo importante. (30)

Dewes (6) sugiere que el proceso enfermero (valoración, diagnóstico, planificación, ejecución y evaluación) es aplicable tanto para cuidar de personas como para cuidar de los datos que genera. Esto debe ser tenido en cuenta a la hora de evaluar la evolución del rol de la enfermería, ya que como hemos visto hay una relación que tiende a ser cada vez más directa entre ambos cuidados.

La automatización de la recogida e interpretación de datos clínicos permite el desarrollo de tecnologías predictivas, útiles para la toma de decisiones. Surgen paralelismos con el salto de la etapa productiva artesanal al trabajo fabril manufacturero durante la Revolución Industrial: de manera casi imperceptible, son las máquinas las que pasan a necesitar a las personas para continuar con la producción, y no al revés.
Si bien parece improbable que estos avances desplacen a los trabajadores durante el acto quirúrgico, es esperable una mejora considerable de los resultados clínicos en los postoperatorios debido al perfeccionamiento de los cuidados técnicos. Es posible que, en unidades postoperatorias, Enfermería corra el riesgo de ver reducidas sus plantillas sin afectación de la calidad de los cuidados, debido al aumento del rendimiento y de la productividad de los hospitales como empresas de servicios, ya sean públicos o privados.

Es por ello por lo que la profesión no debe conformarse con hacer lo mismo que hasta ahora. Es momento de que Enfermería reivindique más que nunca su papel en la gestión y en la investigación, así como en la atención de las necesidades psicosociales, que en ocasiones quedan en un segundo plano. Son precisamente las necesidades que nunca podrán cubrir un algoritmo o un dispositivo. El tiempo de trabajo asistencial liberado por la tecnología que no se traduzca en la potenciación de otros roles, y en la mejora del cuidado de la esfera psicosocial, correrá en contra de los pacientes y de la propia profesión a largo plazo, ya que se convertirá en desempleo.

La revolución tecnológica en la que nos estamos adentrando rápidamen- 
te no debe ser vista como una amenaza. La enfermería como ciencia seguirá teniendo su objeto de estudio: el cuidado humano. Y donde hay personas y comunidades, siempre habrá necesidades por cubrir.

\section{CONCLUSIÓN}

Las innovaciones más recientes en el entorno quirúrgico suponen un salto cualitativo en la mejora del rendimiento $y$ de la calidad de los cuidados, sobre todo gracias a la recogida masiva y posterior análisis de los datos que generan las estancias hospitalarias. Esto permite la optimización de los recursos materiales y humanos, y la mejora de la seguridad de los pacientes y de las comunicaciones entre los miembros de los equipos sanitarios, y entre estos y los familiares de los usuarios.

En este nuevo contexto, la enfermería de quirófano es consciente de que la experiencia quirúrgica para el paciente va a sufrir cambios sustanciales, pero no parece tener una opinión unánime sobre su propio futuro.

\section{AGRADECIMIENTOS}

Quisiera agradecer la orientación metodológica de la doctora Dolores Torres Enamorado (Departamento de Enfermería, Hospital San Juan de Dios de
Bormujos, Sevilla), que aportó a este trabajo mayor coherencia y solidez. 


\section{BiBLIOGRAFÍA}

1. Nightingale F. Notas sobre Enfermeria: Qué es y qué no es. Masson. Barcelona: Dover Publications; 1995. $168 \mathrm{p}$.

2. Rifkin J. La tercera revolución industrial. Barcelona: Paidós; 2011. 393 p.

3. Rifkin J. La sociedad de coste marginal cero: el internet de las cosas, los bienes comunes y el eclipse del capitalismo. Barcelona: Paidós; 2014. $464 \mathrm{p}$.

4. Stanton C. Perspectives: A Window into the World of OR Technology. AORN J. 2015;102(3):2335.

5. Sipes C, Baker JD. Technology in the OR: AORN Members' Perceptions of the Effects on Workflow Efficiency and Quality Patient Care. AORN J. 2015 Sep;102(3):289.e1-19.

6. Dewes A. Improving Performance Measures With Perioperative Analytics. AORN J. 2018 Feb; 107(2):189-98.

7. Azzi AJ, Shah K, Seely A, Villeneuve JP, Sundaresan SR, Shamji FM, et al. Surgical team turnover and operative time: An evaluation of operating room efficiency during pulmonary resection. J Thorac Cardiovasc Surg. 2016 May;151(5):1391-5.

8. Guédon ACP, Paalvast M, Meeuwsen FC, Tax DMJ, van Dijke AP, Wauben LSGL, et al. 'It is Time to Prepare the Next patient' Real-Time Prediction of Procedure Duration in Laparoscopic Cholecystectomies. J Med Syst. 2016 Dec;40(12):271.

9. Kranzfelder M, Schneider A, Fiolka A, Schwan $E$, Gillen $S$, Wilhelm D, et al. Real-time instrument detection in minimally invasive surgery using radiofrequency identification technology. J Surg Res. 2013 Dec;185(2):704-10.

10. McGlothlin JD, Moenning JE, Cole SS. Evaluation and control of waste anesthetic gases in the postanesthesia care unit. J perianesthesia Nurs Off J Am Soc PeriAnesthesia Nurses. 2014 Aug; 29(4):298-312.

11. Kloeze C, Klompenhouwer EG, Brands PJM, van Sambeek MRHM, Cuypers PWM, Teijink JAW. Editor's choice--Use of disposable radiationabsorbing surgical drapes results in significant dose reduction during EVAR procedures. Eur $\mathrm{J}$ Vasc Endovasc Surg. 2014 Mar;47(3):268-72.

12. Weller J, Cumin D, Torrie J, Boyd M, Civil I, Madell $\mathrm{D}$, et al. Multidisciplinary operating room simulation-based team training to reduce treatment errors: a feasibility study in New Zealand hospitals. N Z Med J. 2015 Aug;128(1418):40-51.

13. Glaser B, Schellenberg T, Koch L, Hofer M, Modemann S, Dubach P, et al. Design and evaluation of an interactive training system for scrub nurses. Int J Comput Assist Radiol Surg. 2016 Aug;11(8):152736.
14. Van Gent J-M, Davis KL, Henry N, Zander AL, Kuettel MA, Edson T, et al. The Initial Impact of Tele-Critical Care on the Surgical Services of a Community Military Hospital. Mil Med. 2018 Apr;

15. Nadeau S, Larson S, Bennick V, Bergan T, Martin L, Senst K, et al. Development of a Formalized Pediatric Visitation Program in the PACU Through Evidence-Based Practice. J perianesthesia Nurs Off J Am Soc PeriAnesthesia Nurses. 2016 Feb;31(1): 73-82.

16. Burke-Aaronson AC. Skin-to-skin care and breastfeeding in the perioperative suite. MCN Am J Matern Child Nurs. 2015;40(2):105-9.

17. Wieck MM, Blake B, Sellick C, Kenron D, DeVries D, Terry S, et al. Utilizing technology to improve intraoperative family communication. Am J Surg. 2017 May;213(5):895-900.

18. Hansen M. Text With Surgery Patients - A Research Hypothesis in Enhancing Education and Physical Assessment for Abdominal Surgical Patients. Stud Health Technol Inform. 2016;225:500-4.

19. Odom-Forren J. Perioperative Surgical Home-An Innovative Concept. J Perianesth Nurs. 2016;31(2):109-11.

20. Esser J, Shrinski K, Cady R, Belew J. Reducing OR Traffic Using Education, Policy Development, and Communication Technology. AORN J [Internet]. 2016 Jan;103(1):82-8. Available from: http://search.ebscohost.com/login.aspx? direct $=$ true $\& d b=r z h \& A N=112345522 \&$ site $=$ ehost live\&scope $=$ site

21. Tepas JJ 3rd, Rimar JM, Hsiao AL, Nussbaum MS. Automated analysis of electronic medical record data reflects the pathophysiology of operative complications. Surgery. 2013 Oct;154(4):916-8.

22. Levchenko Al, Boscart VM, Fernie GR. The effect of automated monitoring and real-time prompting on nurses' hand hygiene performance. Comput Inform Nurs. 2013 Oct;31(10):498-504.

23. Soysal GE, Ilce A, Erkol MH. Effect of "An Innovative Technology" Active Warming and Passive Warming on Unplanned Hypothermia During Perioperative Period: A Clinical Trial. Ther Hypothermia Temp Manag. 2018 May;

24. Freitas PS, Silveira RC de CP, Clark AM, Galvao CM. Surgical count process for prevention of retained surgical items: an integrative review. J Clin Nurs. 2016 Jul;25(13-14):1835-47.

25. Schnock KO, Biggs B, Fladger A, Bates DW, Rozenblum R. Evaluating the Impact of Radio Frequency Identification Retained Surgical Instruments Tracking on Patient Safety: Literature Review. J Patient Saf. 2017 Feb;

26. Rao AD, Kumar A, McHugh M. Better Nurse Autonomy Decreases the Odds of 30-Day Mortality and Failure to Rescue. J Nurs Scholarsh an Off Publ Sigma Theta Tau Int Honor Soc Nurs. 2017 Jan; 49(1):73-9. 
27. Luck ES, Gillespie BM. Technological Advancements in the OR: Do We Need to Redefine Intraoperative Nursing Roles? AORN J. 2017 Oct; 106(4):280-2.

28. Chard R. The personal and professional impact of the future of nursing report. AORN J. 2013 Sep;98(3):273-80.

29. Special report: the future of perioperative practice. AORN J. 2017 Feb;105(2):P6-15.
30. Byrne MD. Engaged: the potentially rocky marriage of patients and their digital data. J Perianesth Nurs. 2014;29(3):242-5.

(26) Castro T, Schleder L (2017). The use of gamifi cation to teach in the nursing field. Rev Bras Enferm. 2018;71(3): p. 1038-45.

(27) Orkaizagirre A, Amezcua M, Huércanos L, Arroyo A. El Estudio de casos, un instrumento de aprendizaje en la Relación de Cuidado. Index Enferm. 2014; 23 (4): p. 244-249.

\section{ANEXOS Y TABLAS}

Tabla 3: Resumen de los artículos incluidos en la revisión

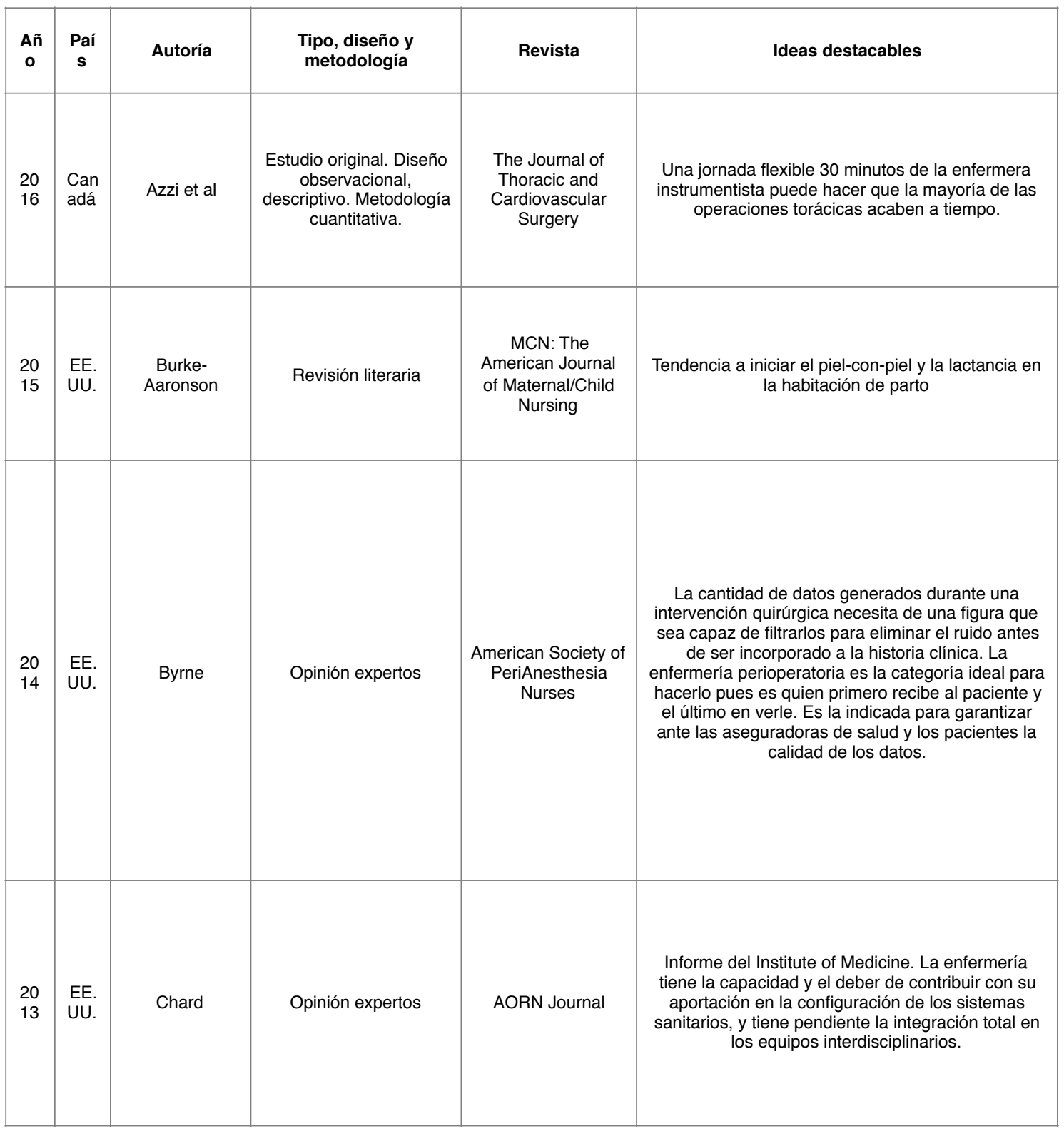




\begin{tabular}{|c|c|c|c|c|c|}
\hline $\begin{array}{l}20 \\
18\end{array}$ & $\begin{array}{l}\text { EE. } \\
\text { UU. }\end{array}$ & Dewes & Opinión expertos & AORN Journal & $\begin{array}{l}\text { Mejorar la eficiencia a través del análisis de datos. } \\
\text { Dato aproximado: } \$ 15-20 / \text { min/operación. Nombra } \\
\text { los indicadores clave para evaluar la calidad. Uso } \\
\text { de datos para gestionar el bloque quirúrgico } \\
\text { identificando problemas y reasignando recursos } \\
\text { para programar las intervenciones. Propone uso del } \\
\text { proceso enfermero en análisis de datos. }\end{array}$ \\
\hline $\begin{array}{l}20 \\
16\end{array}$ & $\begin{array}{l}\text { EE. } \\
\text { UU. }\end{array}$ & Esser et al & $\begin{array}{l}\text { Estudio original. Diseño } \\
\text { experimental. Ensayo } \\
\text { de campo. Metodología } \\
\text { Cuantitativa. }\end{array}$ & AORN Journal & $\begin{array}{l}\text { Programa de reducción de desplazamientos en } \\
\text { quirófano para evitar, en última instancia, } \\
\text { infecciones de heridas quirúrgicas. }\end{array}$ \\
\hline $\begin{array}{l}20 \\
16\end{array}$ & $\begin{array}{l}\text { Bra } \\
\text { sil }\end{array}$ & Freitas et al & $\begin{array}{l}\text { Revisión en PubMed, } \\
\text { CINAHL and } \\
\text { LILACS }\end{array}$ & $\begin{array}{l}\text { Journal of Clinical } \\
\text { Nursing }\end{array}$ & $\begin{array}{l}\text { Diferentes tecnologías para el recuento de gasas: } \\
\text { rayos } X \text {, radio frecuencia y códigos de barras. } \\
\text { Ninguna tecnología sustituye el contaje manual. } \\
\text { Las etiquetas de RF han dado problemas usadas } \\
\text { en el instrumental quirúrgico. }\end{array}$ \\
\hline $\begin{array}{l}20 \\
16\end{array}$ & $\begin{array}{l}\text { Ale } \\
\text { man } \\
\text { ia }\end{array}$ & Glaser et al & $\begin{array}{l}\text { Estudio original. Diseño } \\
\text { experimental. } \\
\text { Metodología } \\
\text { cuantitativa. }\end{array}$ & $\begin{array}{l}\text { The International } \\
\text { Journal for } \\
\text { Computer Assisted } \\
\text { Radiology and } \\
\text { Surgery }\end{array}$ & $\begin{array}{l}\text { Se utiliza un sistema interactivo para simular el } \\
\text { puesto de trabajo de una enfermera quirúrgica, } \\
\text { para formar al personal instrumentista. }\end{array}$ \\
\hline $\begin{array}{l}20 \\
16\end{array}$ & $\begin{array}{l}\text { Paí } \\
\text { ses } \\
\text { Baj } \\
\text { os }\end{array}$ & Guedon et al & $\begin{array}{l}\text { Estudio original. Diseño } \\
\text { observacional, analítico. } \\
\text { Metodología } \\
\text { cuantitativa. }\end{array}$ & $\begin{array}{l}\text { Journal of Medical } \\
\text { Systems }\end{array}$ & $\begin{array}{l}\text { Desarrollo de un sistema de predicción de fin de } \\
\text { cirugía, conectado con el personal encargado de la } \\
\text { programación de los quirófanos. Se utiliza un solo } \\
\text { indicador: el uso de electrobisturí. Mejora la gestión } \\
\text { del uso de los quirófanos. }\end{array}$ \\
\hline $\begin{array}{l}20 \\
16\end{array}$ & $\begin{array}{l}\text { EE. } \\
\text { UU. }\end{array}$ & Hansen & $\begin{array}{l}\text { Estudio original. Diseño } \\
\text { experimental, ensayo de } \\
\text { campo. Metodología } \\
\text { cuantitativa. }\end{array}$ & Nursing Informatics & $\begin{array}{l}\text { Contacto con los pacientes en pre y post cirugía } \\
\text { para mejorar resultados quirúrgicos (sin resultado } \\
\text { aún). }\end{array}$ \\
\hline
\end{tabular}




\begin{tabular}{|c|c|c|c|c|c|}
\hline $\begin{array}{l}20 \\
14\end{array}$ & $\begin{array}{l}\text { Paí } \\
\text { ses } \\
\text { Baj } \\
\text { os }\end{array}$ & Kloeze et al & $\begin{array}{l}\text { Estudio original. Diseño } \\
\text { experimental ensayo } \\
\text { clínico. Metodología } \\
\text { cuantitativa. }\end{array}$ & $\begin{array}{l}\text { European Journal } \\
\text { of Endovascular } \\
\text { Surgery }\end{array}$ & $\begin{array}{l}\text { Uso de paños quirúrgicos desechables que } \\
\text { absorben la expansión de la radiación en } \\
\text { reparaciones aórticas endovasculares. Reduce la } \\
\text { exposición de los sanitarios entre } 48 \text { y } 55 \% \text {. }\end{array}$ \\
\hline $\begin{array}{l}20 \\
13\end{array}$ & $\begin{array}{c}\text { Ale } \\
\text { man } \\
\text { ia }\end{array}$ & $\begin{array}{l}\text { Kranzfelder et } \\
\text { al }\end{array}$ & $\begin{array}{l}\text { Estudio original. Diseño } \\
\text { analítico, experimental. } \\
\text { Metodología } \\
\text { cuantitativa. }\end{array}$ & $\begin{array}{l}\text { Journal of Surgical } \\
\text { Research }\end{array}$ & $\begin{array}{l}\text { Monitorización a tiempo real del instrumental en } \\
\text { uso en un quirófano con tecnología RFID. }\end{array}$ \\
\hline $\begin{array}{l}20 \\
13\end{array}$ & $\begin{array}{l}\text { Can } \\
\text { adá }\end{array}$ & $\begin{array}{l}\text { Levchenko et } \\
\text { al }\end{array}$ & $\begin{array}{l}\text { Estudio original. Diseño } \\
\text { analítico, experimental. } \\
\text { Ensayo de campo. } \\
\text { Metodología } \\
\text { cuantitativa. }\end{array}$ & $\begin{array}{l}\text { CIN: Computers, } \\
\text { Informatics, Nursing }\end{array}$ & $\begin{array}{l}\text { Sistema de monitorización a tiempo real del estado } \\
\text { de higiene de las manos, capaz de detectar los } \\
\text { momentos ideales para el lavado. }\end{array}$ \\
\hline $\begin{array}{l}20 \\
17\end{array}$ & $\begin{array}{l}\text { EE. } \\
\text { UU. }\end{array}$ & $\begin{array}{l}\text { Luck + } \\
\text { Gillespie }\end{array}$ & $\begin{array}{l}\text { Editorial. } \\
\text { Opinión. }\end{array}$ & AORN Journal & $\begin{array}{l}\text { Sobre el progresivo cambio en el rol de Enfermería } \\
\text { debido a los cambios tecnológicos: cirugía robótica, } \\
\text { plataformas de simulación, imágenes a tiempo real } \\
\text { en el campo quirúrgico y "zonas de navegación" } \\
\text { con código de colores para distinguir áreas } \\
\text { neoplásicas. Fantasea con cintas de transporte } \\
\text { para pacientes y la sustitución casi total del } \\
\text { personal de quirófano. Sugiere una futura } \\
\text { disminución de la necesidad de anticipación de las } \\
\text { enfermeras instrumentistas, y un cambio de rol } \\
\text { orientado hacia lo técnico. }\end{array}$ \\
\hline $\begin{array}{l}20 \\
14\end{array}$ & $\begin{array}{l}\text { EE. } \\
\text { UU. }\end{array}$ & $\begin{array}{l}\text { McGlothlin + } \\
\text { Cole }\end{array}$ & $\begin{array}{l}\text { Estudio original. Diseño } \\
\text { analítico, observacional, } \\
\text { caso-control. } \\
\text { Metodología } \\
\text { cuantitativa. }\end{array}$ & $\begin{array}{l}\text { American Society of } \\
\text { PeriAnesthesia } \\
\text { Nurses }\end{array}$ & $\begin{array}{l}\text { Uso de infrarrojos para detectar gases anestésicos } \\
\text { en URPA. }\end{array}$ \\
\hline $\begin{array}{l}20 \\
16\end{array}$ & $\begin{array}{l}\text { EE. } \\
\text { UU. }\end{array}$ & Nadeau et al & $\begin{array}{l}\text { Estudio original. Diseño } \\
\text { observacional, } \\
\text { descriptivo, transversal. } \\
\text { Metodología } \\
\text { cuantitativa. }\end{array}$ & $\begin{array}{l}\text { American Society of } \\
\text { PeriAnesthesia } \\
\text { Nurses }\end{array}$ & $\begin{array}{l}\text { Programa de visitas a la URPA que se implanta } \\
\text { satisfactoriamente. }\end{array}$ \\
\hline
\end{tabular}




\begin{tabular}{|c|c|c|c|c|c|}
\hline $\begin{array}{l}20 \\
16\end{array}$ & $\begin{array}{l}\text { EE. } \\
\text { UU. }\end{array}$ & Odom-Forrem & Editorial. Opinión. & $\begin{array}{l}\text { Journal of } \\
\text { PeriAnesthesia } \\
\text { Nursing }\end{array}$ & $\begin{array}{l}\text { Surgimiento del modelo Perioperative Surgical } \\
\text { Home y del papel que los enfermeros } \\
\text { perioperatorios de practica avanzada tienen en su } \\
\text { desarrollo. }\end{array}$ \\
\hline $\begin{array}{l}20 \\
16\end{array}$ & $\begin{array}{l}\text { EE. } \\
\text { UU. }\end{array}$ & Rao et al & $\begin{array}{l}\text { Estudio original. Diseño } \\
\text { analítico, observacional. } \\
\text { Metodología } \\
\text { cuantitativa. }\end{array}$ & $\begin{array}{l}\text { Journal of Nursing } \\
\text { Scholarship }\end{array}$ & $\begin{array}{l}\text { El fomento de la autonomía enfermera mejora el } \\
\text { indicador de mortalidad a los } 30 \text { días. }\end{array}$ \\
\hline $\begin{array}{l}20 \\
17\end{array}$ & $\begin{array}{l}\text { EE. } \\
\text { UU. }\end{array}$ & Schnock et al & Revisión literaria & $\begin{array}{l}\text { Journal of Patient } \\
\text { Safety }\end{array}$ & $\begin{array}{l}\text { Tecnología RFID reduce los errores quirúrgicos por } \\
\text { retención de instrumental. }\end{array}$ \\
\hline $\begin{array}{l}20 \\
15\end{array}$ & $\begin{array}{l}\text { EE. } \\
\text { UU. }\end{array}$ & Sipes + Baker & Opinión expertos & AORN Journal & $\begin{array}{c}\text { Percepción de los miembros de la AORN sobre las } \\
\text { nuevas tecnologías en quirófano. }\end{array}$ \\
\hline $\begin{array}{l}20 \\
18\end{array}$ & $\begin{array}{l}\text { Tur } \\
\text { quía }\end{array}$ & Soysal et al & $\begin{array}{l}\text { Estudio original. Diseño } \\
\text { observacional, analítico, } \\
\text { caso-control. } \\
\text { Metodología } \\
\text { cuantitativa. }\end{array}$ & $\begin{array}{l}\text { Therapeutic } \\
\text { Hypothermia And } \\
\text { Temperature } \\
\text { Management }\end{array}$ & $\begin{array}{l}\text { Uso de fibra de carbón para calentamiento activo } \\
\text { en quirófano. Mejor que calentamiento pasivo. }\end{array}$ \\
\hline $\begin{array}{l}20 \\
15\end{array}$ & $\begin{array}{l}\text { EE. } \\
\text { UU. }\end{array}$ & Stanton & Editorial. Opinión. & AORN Journal & $\begin{array}{l}\text { Clasifica los nuevos avances en dos categorías: } \\
\text { Eficiencia en el Flujo de Trabajo y Calidad de los } \\
\text { Cuidados del Paciente. }\end{array}$ \\
\hline $\begin{array}{l}20 \\
13\end{array}$ & $\begin{array}{l}\text { EE. } \\
\text { UU. }\end{array}$ & Tepas et al & $\begin{array}{l}\text { Estudio original. Diseño } \\
\text { analítico, observacional. } \\
\text { Metodología } \\
\text { cuantitativa. }\end{array}$ & Surgery Journal & $\begin{array}{l}\text { Desarrollo de un algoritmo que utiliza información } \\
\text { de la historia clínica digital y anticipa eventos que, } \\
\text { de ser atendidos, podría reducirse la mortalidad en } \\
\text { casos de sepsis. }\end{array}$ \\
\hline $\begin{array}{l}20 \\
18\end{array}$ & $\begin{array}{l}\text { EE. } \\
\text { UU. }\end{array}$ & VanGent et al & $\begin{array}{l}\text { Estudio original. Diseño } \\
\text { experimental. } \\
\text { Intervención. } \\
\text { Metodología } \\
\text { cuantitativa. }\end{array}$ & Military Medicine & $\begin{array}{c}\text { Modelo de telemedicina que permite la asistencia } \\
\text { de un intensivista a distancia en pacientes críticos } \\
\text { postquirúrgicos, }\end{array}$ \\
\hline $\begin{array}{l}20 \\
15\end{array}$ & $\begin{array}{c}\text { N. } \\
\text { Zela } \\
\text { nda }\end{array}$ & Weller et al & $\begin{array}{l}\text { Estudio original. Diseño } \\
\text { experimental. } \\
\text { Metodología } \\
\text { cuantitativa. }\end{array}$ & $\begin{array}{l}\text { New Zealand } \\
\text { Medical Journal }\end{array}$ & $\begin{array}{l}\text { Estudio que cuantifica el coste de mejorar la } \\
\text { comunicación intraequipo en quirófano con } \\
\text { simulaciones realistas. }\end{array}$ \\
\hline
\end{tabular}




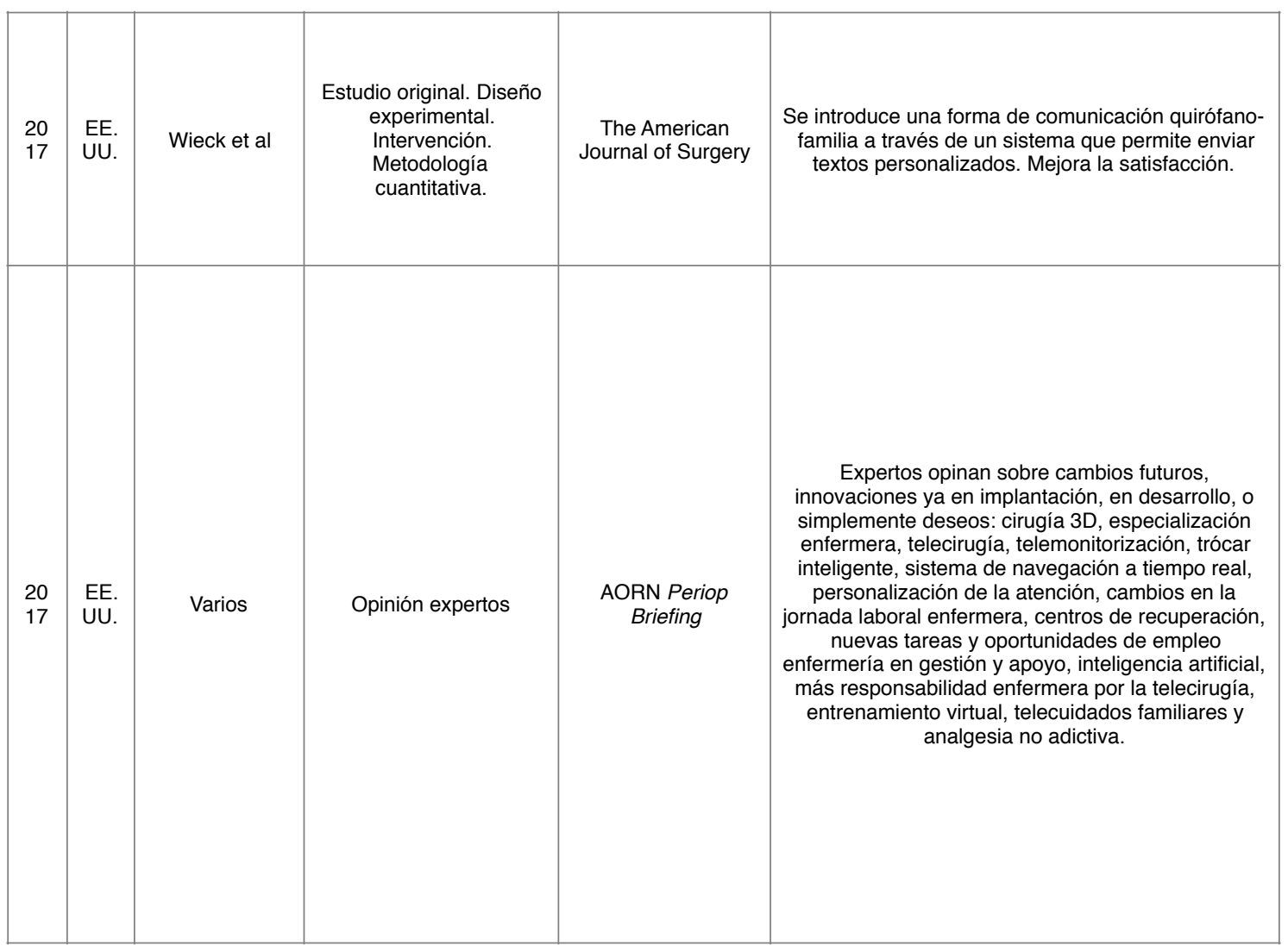

